\title{
Diffusivity Control of Heat Transfer Process Using Optimality Conditions
}

\author{
Seda Göktepe Körpeoğlu ${ }^{1^{*}}$ \\ 1* Yildiz Technical University, Faculty of Chemical and Metallurgical Engineering, Departmant of Mathematical Engineering, İstanbul, Turkey, (ORCID: 0000-0001- \\ 7146-0846), sgoktepe@yildiz.edu.tr
}

(First received 5 April 2021 and in final form 27 June 2021)

(DOI: $10.31590 /$ ejosat.909910)

\begin{abstract}
ATIF/REFERENCE: Göktepe Körpeoğlu, S. (2021). Diffusivity Control of Heat Transfer Process Using Optimality
\end{abstract} Conditions. European Journal of Science and Technology, (25), 341-346.

\begin{abstract}
In this paper, a distributed parameter system expressed as a parabolic partial differential equation governed by a diffusivity control is considered. A modal space expansion approach is used to convert the distributed parameter system into a lumped parameter system. Thereafter, Pontryagin's maximum principle is used to compute the optimal control function that leads to a nonlinear two-point boundary value problem (TPBVP). An iterative numerical technique, variation of extremals is used to solve the nonlinear TPBVP. The feasibility and applicability of the proposed solution is demonstrated by numerical simulations generated in MATLAB.
\end{abstract}

Keywords: Diffusivity control, Maximum principle, Parabolic PDE.

\section{Optimallik Koşulları Kullanılarak Isı Transferi İşleminin Yayılım Kontrolü}

$\ddot{\mathbf{O z}}$

Bu çalışmada, yayılım kontrolü ile yönetilen parabolik kısmi diferansiyel denklem olarak ifade edilen dağıtılmış bir parametre sistemi ele alınmıştır. Dağıtılmış parametre sistemini toplu bir parametre sistemine dönüştürmek için bir özfonksiyon genişletme yaklaşımı kullanılmıştır. Bundan sonra, Pontryagin'in maksimum prensibi, doğrusal olmayan iki noktalı sınır değeri problemine yol açan optimum kontrol fonksiyonunu hesaplamak için kullanılmıştır. Doğrusal olmayan iki noktalı sınır değer problemini çözmek için, yinelemeli sayısal bir teknik, variation of extremals, kullanılmış̧ır. Önerilen çözümün fizibilitesi ve uygulanabilirliği, MATLAB'da oluşturulan sayısal simülasyonlarla gösterilmiştir.

Anahtar Kelimeler: Yayılım kontrolü, Maksimum Prensibi, Parabolik KDD.

\footnotetext{
*Corresponding Author: sgoktepe@yildiz.edu.tr
} 


\section{Introduction}

Parabolic equations can be used to model a variety of dissipative physical processes. In the field of distributed parameter system theory, there is a long tradition of study into the related control problems ( $\mathrm{Xu}$ et al., 2011). Boundary conditions (boundary control), source terms (interior control) and diffusivity coefficients (diffusivity control) are some examples of physical actuation in parabolic partial differential equations (PDEs). Interior and boundary control issues have been thoroughly researched. However, controlling of PDEs using a diffusivity actuator has rarely been studied. Lin et al. discussed a parabolic system with a bilinear control and they proved the exact controllability of the system (Lin et al., 2007a). Xu et al. studied a controlled parabolic system with diffusivity, boundary and interior actuations. The solution of the reduced-order system obtained is computed with a successive scheme based on Picard apparoximation (Xu et al., 2007). Lin et al. investigated a nonlinear parabolic structure controlled by a bilinear control and an approximate null controllability result is presented (Lin et al., $2007 \mathrm{~b}$ ). The parabolic system's nonnegative approximate controllability is researched through a bilinear control (Khapalov, 2003). Körpeoğlu and Küçük introduced an approach containing modal space expansion, Pontryagin's maximum principle and steepest descent algorithm to solve a parabolic bilinear optimal control problem (Körpeoğlu and Küçük, 2018).

There are three distinct classes of solution methods for optimal control problems: Dynamic programming, direct methods and indirect methods. The method of dynamic programming leads to a nonlinear partial differential equation such as HamiltonJacobi-Bellman equations. In direct methods, calculus of variations, mathematical programming and state and/or control parametrization, optimal control problems are transformed into nonlinear optimization problems. As for an indirect method, Pontryagin's maximum principle is used to derive the necessary conditions for optimality. The obtained optimality conditions result in nonlinear two-point boundary-value problems. The solutions of TPBVPs are computed via iterative numerical solution techniques such as steepest descent, quasilinearization and variation of extremals methods (Kirk, 2004).

In this paper, it is aimed to obtain the optimal control function to a parabolic equation with a diffusivity control where a heat conduction process is studied as the model problem. Controlling the dynamic response of the system by using an accessible control is indicated by a quadratic performance index to be minimized. An indirect method based on Pontryagin's maximum principle is used to derive necessary conditions for the optimal control. This approach leads to a nonlinear two-point boundary value problem with split boundary conditions. The variation of extremals method is used as a solution procedure. Numerical calculations illustrate the theoretical approach's efficacy and applicability.

The paper is organized as follows. The optimal control problem formulation and uniqueness and controllability of the problem are examined in Section 2. In Section 3, modal control space problem is discussed to obtain reduced order model. In Section 4, derivation of the Pontryagin's maximum principle for the controlled system is introduced. A simulation study is presented in Section 5 using the variation of extremals method with numerical results to verify the competence of the introduced control algorithm. Section 6 contains the results and discussion. The paper's conclusion is stated in Section 7.

\section{Optimal Control Problem}

\subsection{Problem Formulation}

Consider a parabolic distributed parameter system that describes a heat transfer mechanism (Lin et al., 2007a) defined by the following relationship

$$
\begin{aligned}
& \frac{\partial z}{\partial t}=\Delta z+\chi_{\omega} u(t)(z-\xi(x, t)), \\
& z(0, t)=z_{B}{ }^{(0)}(t), \quad z(l, t)=z_{B}{ }^{(l)}(t), \\
& z(x, 0)=\varphi(x),
\end{aligned}
$$

over $\Omega=\left\{(x, t): 0 \leq x \leq l, t_{0} \leq t \leq t_{f}\right\} \cdot \chi_{\omega}$ is the characteristic function of $\omega, u(t)$ is the control function to be determined optimally. $\Omega_{t}$ denotes a given time interval $\left(0, t_{f}\right)$ where $t_{f}$ is a predetermined terminal time. $\varphi(x)$ is the initial profile and $z_{B}{ }^{(0)}(t), z_{B}{ }^{(l)}(t)$ are given functions on $\Omega_{t}$. The term $u(t)(z-$ $\xi(x, t))$ denotes the heat-exchange of the given substance at system at position $x$ and time $t$ with the sorrounding medium of the temperature $\xi(x, t)$. The control function $u(t)$ is used as a catalysts that can accelerate or decelerate the reaction.

The following perfomance index functional is used to formulate the optimal control problem for the parabolic system (1)-(3).

$$
\begin{gathered}
\min _{u} J=\frac{1}{2} \int_{0}^{l}\left\langle z_{t_{f}}(x), \mathcal{P}_{z_{t_{f}}}(x)\right\rangle d x+\frac{1}{2} \int_{\Omega}\langle z(x), \mathcal{R} z(x)\rangle d x d t \\
+\frac{1}{2} \int_{t_{0}}^{t_{f}}\langle\mathcal{S} u, u\rangle d t
\end{gathered}
$$

where $\mathcal{P}$ and $\mathcal{R}$ are weighting operators, $\mathcal{S}$ is the control weighting matrix. $z\left(x, t_{f}\right)=z_{t_{f}}(x)$ is the evaluated state at time $t=t_{f}$. The following functional space is defined with the inner product $\langle.,$.$\rangle ,$

$$
\begin{aligned}
& L^{2}(0, l)=\left\{f(x): \int_{\Omega} f^{2}(x) d x<\infty\right\}, \\
& H^{1}(0, l)=\left\{f: f \in L^{2}(0, l) \text { and } f^{\prime} \in L^{2}\right\} .
\end{aligned}
$$

Presume that the control set that is admissible is

$$
U_{a d}=\left\{u(t): u(t) \in L^{2}\left(\Omega_{t}\right)\right\} .
$$

Finding an optimal control function $u^{*}(t) \in U_{a d}$ that minimizes the performance index is required.

\subsection{Uniqueness of the Solution}

The existence of the solution for the optimal control problem (1)-(3) is considered in (Lin et al., 2007). The uniqueness of the problem is then illustrated next via the application of the energy method. The uniqueness of the system's solution is critical since it determines the control's uniqueness.

Lemma. Eq. (1) has a unique solution $z(x, t) \in L^{2}\left(\mathbb{R}^{N}\right)$ when subjected to Eqs. (2)-(3).

Proof. Assume there are two smooth solutions, $z_{1}$ and $z_{2}$, that satisfy Eq. (1) with $\xi(x, t)=0$. Then, with zero initial-boundary conditions, their difference, $\tilde{z}:=z_{1}-z_{2}$ satisfies Eq. (1).

$$
\begin{aligned}
& \frac{\partial \tilde{z}}{\partial t}=\Delta \tilde{z}+\chi_{\omega} u(t)(\tilde{z}-\xi(x, t)), \\
& \tilde{z}(0, t)=0, \tilde{z}(l, t)=0,
\end{aligned}
$$


$\tilde{z}(x, 0)=0$.

The energy integral is now defined as follows:

$E(\tilde{z})=\frac{1}{2} \int_{0}^{l}|\tilde{z}(x, t)|^{2} d x$

which is always positive and decreasing. Let ||.|| stand for the $L^{2}$ norm on $\mathbb{R}^{N}$ determined by

$$
\|f\|=\left(\int_{\mathbb{R}^{N}}|f(x)|^{2} d x\right)^{1 / 2} .
$$

When you differentiate with respect to $t$ Eq. (11), you get

$$
\begin{aligned}
\frac{d}{d t} E=\int_{0}^{l} \tilde{z} \frac{\partial \tilde{z}}{\partial t} & d x \\
& =\int_{0}^{l} \tilde{z}\left(\Delta \tilde{z}+\chi_{\omega} u(t) \tilde{z}\right) d x \\
& =\int_{0}^{l} \tilde{z} \Delta \tilde{z} d x+\int_{0}^{l} \chi_{\omega} u(t) \tilde{z}^{2} d x
\end{aligned}
$$

Using integrating by parts in the last integral results in

$\frac{d}{d t} E=\tilde{z} \frac{\partial^{2} \tilde{z}}{\partial x^{2}} l_{0}^{l}-\int_{0}^{l}\left(\frac{\partial \tilde{z}}{\partial x}\right)^{2} d x+\int_{0}^{l} \chi_{\omega} u(t) \tilde{z}^{2} d x$,

and we get the following equation

$\frac{d}{d t} E=-\int_{0}^{l}\left(\frac{\partial \tilde{z}}{\partial x}\right)^{2} d x+\chi_{\omega} u(t) \int_{0}^{l} \tilde{z}^{2} d x \leq \chi_{\omega} u(t)\|\tilde{z}\|^{2}$.

Then, we obtain

$\frac{d}{d t}\|\tilde{z}\| \leq \chi_{\omega} u(t)\|\tilde{z}\|^{2}$.

Now we apply Gronwall's lemma, and we get

$\left\|z_{1}-z_{2}\right\| \leq\left|z_{1}-z_{2}\right|_{t=0} \chi_{\omega} \exp \left(\int_{0}^{t_{f}} u(r) d r\right)$.

As a result, the uniqueness of smooth solutions is immediately demonstrated.

To ensure the uniqueness of the solution given by Lemma proposed above, the corresponding control function $u(t)$ must be unique. Since the system has a unique solution and unique control function, the system is called observable. By taking Hilbert Uniqueness into account, the observability is equivalent to the controllability (Guliyev et al., 2007; Pedersen, 1999). Namely, the system is controllable.

\section{Reduced Order Modeling (ROM)}

To convert the optimal control of distributed parameter systems (1)-(3) into the optimal control of lumped parameter systems, the modal space expansion technique is used. As a result, the original parabolic PDE system can be approximated using a low-dimensional dynamical system.

To accomplish the transformation, a new parameter $y(x, t)$ is first applied to translate nonhomogeneous boundary conditions to homogeneous boundary conditions. By introducing

$$
y(x, t)=z(x, t)-\frac{(l-x)}{l} z_{B}{ }^{(0)}(t)-\frac{x}{l} z_{B}{ }^{(l)}(t)
$$
obtained

$$
\begin{aligned}
\frac{\partial y}{\partial t}-\frac{\partial^{2} y}{\partial x^{2}}= & \frac{(x-l)}{l} \frac{\partial\left(z_{B}{ }^{(0)}(t)\right)}{\partial t}-\frac{x}{l} \frac{\partial\left(z_{B}{ }^{(l)}(t)\right)}{\partial t} \\
& +u(t)\left(\chi_{\omega} y(t)-\frac{(l-x)}{l} z_{B}{ }^{(0)}(t)\right. \\
& \left.-\frac{x}{l} z_{B}{ }^{(l)}(t)-\xi(x, t)\right)
\end{aligned}
$$

subject to

$$
\begin{aligned}
& y(x, 0)=\varphi(x)-\frac{(l-x)}{l} z_{B}{ }^{(0)}(0)-\frac{x}{l} z_{B}{ }^{(l)}(0), \\
& y(0, t)=0, y(l, t)=0 .
\end{aligned}
$$

In the following calculations, a truncated Fourier series expansion is used

$$
y(x, t) \approx \sum_{k=1}^{n} \psi_{k}(x) w_{k}(t) .
$$

Indicating a complete orthonormal basis as

$$
V=\left\{v: v, \frac{\partial v}{\partial x} \in L^{2}(\Omega),\left.v\right|_{\partial \Omega}=0\right\}
$$

for $H(0, l)$, the solution $y(x, t)$ of the system is satisfied by multiplying both sides of Eq. (20) by a basis function $v$ and integrating by parts

$$
\begin{aligned}
\int_{0}^{l} \frac{\partial y}{\partial t} v d x-\int_{0}^{l} \frac{\partial^{2} y}{\partial x^{2}} v d x & \\
& =\int_{0}^{l}\left(\frac{(x-l)}{l} \frac{\partial\left(z_{B}{ }^{(0)}(t)\right)}{\partial t}\right. \\
& \left.-\frac{x}{l} \frac{\partial\left(z_{B}{ }^{(l)}(t)\right)}{\partial t}\right) v d x \\
& +\int_{0}^{l} u(t)\left(\chi \omega y(t)-\frac{(l-x)}{l} z_{B}{ }^{(0)}(t)\right. \\
& \left.-\frac{x}{l} z_{B}{ }^{(l)}(t)\right) v d x-\int_{0}^{l} \xi(x, t) v d x
\end{aligned}
$$

where $y, v \in V$. If Eq. (23) for $y(x, t)$ is used in Eq. (25) and $v=$ $\psi_{j}, \mathrm{j}=1,2, \ldots \mathrm{n}$, the finite dimensional system is obtained using the notations below $\left(D:=\frac{\partial}{\partial x}\right)$ :

$$
\frac{d w}{d t}=M w+N w u+K u+L
$$

where $w(t)=\left(w_{1}(t), w_{2}(t), \ldots, w_{n}(t)\right) \in \mathbb{R}^{n}, \quad M=\left(M_{j k}\right) \in$ $\mathbb{R}^{n \times n}, K=K_{j}, L=L_{j} \in \mathbb{R}^{n}$. The finite dimensional approximation is represented by the vector $w(t)$. The initial values are defined by

$$
w_{j}(0)=\left\langle y(x, 0), \psi_{j}\right\rangle .
$$

The following notations are used in Eq. (26):

$$
\begin{aligned}
& F_{j k}=\left\langle\psi_{j}, \psi_{k}\right\rangle=\int_{0}^{l} \psi_{j}(x) \psi_{k}(x) d x=\delta_{j k}, \\
& \delta_{j k}=\left\{\begin{array}{r}
1, \text { if } j=k \\
0, \text { otherwise. }
\end{array}\right. \\
& M_{j k}=\left\langle\psi_{j}, D^{2} \psi_{k}\right\rangle=\int_{0}^{l} \psi_{j}(x) \frac{\partial^{2}\left(\psi_{k}(x)\right)}{\partial x^{2}} d x \\
& N_{j k}=\int_{0}^{l} \chi_{\omega} \psi_{j}(x) d x
\end{aligned}
$$




$$
\begin{aligned}
F_{j} & =\int_{0}^{l}\left(\frac{(x-l)}{l} \frac{\partial\left(z_{B}{ }^{(0)}(t)\right)}{\partial t}-\frac{x}{l} \frac{\partial\left(z_{B}{ }^{(l)}(t)\right)}{\partial t}\right) \psi_{j}(x) d x \\
K_{j} & =\int_{0}^{l}\left(\frac{(l-x)}{l} z_{B}{ }^{(0)}(t)-\frac{x}{l} z_{B}{ }^{(l)}(t)\right) \psi_{j}(x) d x \\
H_{j} & =-\int_{0}^{l} \psi_{j}(x) \xi(x, t) d x \\
L_{j} & =F_{j}+H_{j}
\end{aligned}
$$

\section{Pontryagin's Maximum Principle}

In 1956, Lev Pontryagin, a Russian mathematician, reformulated the control problem in terms of a Hamiltonian. It enables one to express the adjoint equations and optimality conditions in a very compact, general, and simple manner. Pontryagin's method is similar to, and also a generalization of, the classical variational method. To obtain the Euler equations, the classical variational approach requires that all functions, including the state and control variables, be continuously differentiable functions. By relaxing the optimality condition, Pontryagin's approach allows for piecewise-continuous solutions for the control input (Pontryagin, 1959).

The conditions in the Maximum principle are necessary but not sufficient for optimality. We need to look at the control region. $\boldsymbol{U}_{\boldsymbol{a d}}$ must be convex. Then only then $(\boldsymbol{z}(\boldsymbol{t}), \boldsymbol{u}(\boldsymbol{t}))$ is an optimal pair.

The finite horizon optimal control problem defined in Eq. (4) can now be rewritten as

$$
J=\frac{1}{2}\left[w\left(t_{f}\right)\right]^{T} P\left[w\left(t_{f}\right)\right]+\frac{1}{2} \int_{t_{0}}^{t_{f}}\left(w^{T} R w+u^{T} S u\right) d t
$$

$\mathrm{P}, \mathrm{R}$ and $\mathrm{S}$ are symmetric positive semi-definite matrices which are the finite dimensional representations of $\mathcal{P}, \mathcal{R}$ and $\boldsymbol{S}$.

Theorem. A canonical optimality condition is obtained, which is a nonlinear two-point boundary value problem by using the Pontryagin's Maximum Principle

$$
\left\{\begin{array}{c}
\frac{d w}{d t}=M w-S^{-1} \Lambda^{T}(N w+K)^{2}+L \\
\frac{d \Lambda}{d t}=-R w-M^{T} \Lambda-N u^{T} \Lambda \\
\Lambda\left(t_{f}\right)=P w\left(t_{f}\right) \\
w\left(t_{0}\right)=w_{0}
\end{array}\right.
$$

where $\mathrm{P}, \mathrm{R}$ and $\mathrm{S}$ are positive semidefinite symmetric matrices, M, N, K and L are defined in Eqs. (30), (31), (33) and (35), respectively.

Proof. Consider the optimal control problem of the system Eqs. (26)-(27),

$$
\frac{d w}{d t}=M w+N w u+K u+L
$$

where $w(t)$ is the finite dimensional approximation to $y(x, t)$ and $u(t)$ is the control input. M, N, K and L are defined in Eqs. (30), (31), (33) and (35), respectively.

We minimize the following augmented cost functional,

$$
\begin{aligned}
J^{*}(w, u, \Lambda)= & \int_{t_{0}}^{t_{f}}\left\{\frac{1}{2}\left(w^{T} R w+u^{T} S u\right)\right. \\
& \left.-\Lambda^{T}\left(\frac{d w}{d t}-M w-N w u-K u-L\right)\right\} d t \\
& +\frac{1}{2}\left[w\left(t_{f}\right)\right]^{T} P\left[w\left(t_{f}\right)\right]
\end{aligned}
$$

The functional becomes as following by establishing the Hamiltonian $\mathcal{H}$,

$$
\begin{aligned}
J^{*}(w, u, \Lambda)= & \int_{t_{0}}^{t_{f}}\left\{\mathcal{H}(t, w, u, \Lambda)-\Lambda^{T} \frac{d w}{d t}\right\} d t \\
& +\frac{1}{2}\left[w\left(t_{f}\right)\right]^{T} P\left[w\left(t_{f}\right)\right] .
\end{aligned}
$$

If $(\boldsymbol{w}, \boldsymbol{u}, \boldsymbol{\Lambda})$ is a minimizer of $\boldsymbol{J}^{*}$

$$
\begin{aligned}
\delta J^{*}=\int_{t_{0}}^{t_{f}}\left\{\frac{\partial \mathcal{H}}{\partial w} \delta w\right. & \left.+\frac{\partial \mathcal{H}}{\partial u} \delta u+\frac{\partial \mathcal{H}}{\partial \Lambda} \delta \Lambda-\delta\left(\Lambda^{T} \frac{d w}{d t}\right)\right\} d t \\
& +\delta\left(\frac{1}{2}\left[w\left(t_{f}\right)\right] P\left[w\left(t_{f}\right)\right]\right) \\
& =0 .
\end{aligned}
$$

We get the following four optimality conditions after processing variational operations and integration by parts:

Firstly,

$$
\begin{aligned}
& \frac{\partial \mathcal{H}}{\partial u}=0 \\
& u(t)=-S^{-1} \Lambda^{T}(N w+K)
\end{aligned}
$$

Secondly,

$$
\begin{aligned}
& \frac{\partial \mathcal{H}}{\partial w}=-\frac{d \Lambda}{d t} \\
& \frac{d \Lambda}{d t}=-R w-M^{T} \Lambda-N u^{T} \Lambda
\end{aligned}
$$

Thirdly,

$$
\begin{aligned}
& \frac{\partial \mathcal{H}}{\partial \Lambda}=\frac{d \mathrm{w}}{d t} \\
& \frac{d \mathrm{w}}{d t}=M w-S^{-1} \Lambda^{T}(N w+K)^{2}+L
\end{aligned}
$$

Lastly,

$$
P w\left(t_{f}\right)=\Lambda\left(t_{f}\right)
$$

To obtain the control law in this proof, the Pontryagin's approach leads to a nonlinear two-point boundary-value problem that cannot be solved analytically. The combination of split boundary conditions and nonlinear differential equations makes solving the optimal control problem challenging. On a numerical example, an iterative numerical technique for evaluating optimal control and trajectories is discussed in the following section. Variation of extremals method is used as a numerical method.

\section{Algorithm Based on Variation of Extremals}

The following procedure explains how the variation of extremals method works to solve the nonlinear two-point boundary value problem (Kirk, 2004). The method of variation of 
extremals is an algorithm that uses the observed values of $\boldsymbol{\Lambda}\left(\boldsymbol{t}_{\boldsymbol{f}}\right)$ to adjust systematically the guessed values of $\boldsymbol{\Lambda}\left(\boldsymbol{t}_{\mathbf{0}}\right)$. Newton's method is used for making systematic adjustments of the initial costate values.

Let's start with an overview of the steps involved in using the variation of extremals method:

1. Solve $\frac{\partial \mathcal{H}}{\partial \boldsymbol{u}}=\mathbf{0}$ for $\boldsymbol{u}(\boldsymbol{t})$ in terms of $\boldsymbol{w}(\boldsymbol{t}), \boldsymbol{\Lambda}(\boldsymbol{t})$, and substitute in the state and costate equations to produce the reduced differential equations.

2. Set the iteration index $i$ to zero and guess $\boldsymbol{\Lambda}^{\mathbf{0}}\left(\boldsymbol{t}_{\mathbf{0}}\right)$, the costate's initial value.

3. Integrate the reduced state-costate equations and the influence function equations (45) and (46) with initial conditions (47) and (48), from $\boldsymbol{t}_{\mathbf{0}}$ to $\boldsymbol{t}_{f}$, using $\boldsymbol{\Lambda}\left(\boldsymbol{t}_{\mathbf{0}}\right)=\boldsymbol{\Lambda}^{\boldsymbol{i}}\left(\boldsymbol{t}_{\mathbf{0}}\right)$ and $\boldsymbol{w}\left(\boldsymbol{t}_{\mathbf{0}}\right)=$ $\boldsymbol{w}_{\mathbf{0}}$ as initial conditions. Only $\boldsymbol{\Lambda}^{i}\left(\boldsymbol{t}_{f}\right), \boldsymbol{w}^{\boldsymbol{i}}\left(\boldsymbol{t}_{f}\right)$, and the state and costate influence matrices $\boldsymbol{P}_{\boldsymbol{\Lambda}}\left(\boldsymbol{\Lambda}^{\boldsymbol{i}}\left(\boldsymbol{t}_{0}\right), \boldsymbol{t}_{f}\right)$ and $\boldsymbol{P}_{w}\left(\boldsymbol{\Lambda}^{\boldsymbol{i}}\left(\boldsymbol{t}_{\mathbf{0}}\right), \boldsymbol{t}_{f}\right)$ should be saved.

$$
\begin{aligned}
& \frac{d}{d t}\left[P_{w}\left(\Lambda^{i}\left(t_{0}\right), t\right)\right]=\left[\frac{\partial^{2} \mathcal{H}}{\partial \Lambda \partial w}(t)\right]_{i} P_{w}\left(\Lambda^{i}\left(t_{0}\right), t\right)+ \\
& {\left[\frac{\partial^{2} \mathcal{H}}{\partial \Lambda^{2}}(t)\right]_{i} P_{\Lambda}\left(\Lambda^{i}\left(t_{0}\right), t\right)} \\
& \frac{d}{d t}\left[P_{\Lambda}\left(\Lambda^{i}\left(t_{0}\right), t\right)\right]=\left[-\frac{\partial^{2} \mathcal{H}}{\partial w^{2}}(t)\right]_{i} P_{w}\left(\Lambda^{i}\left(t_{0}\right), t\right)+ \\
& {\left[-\frac{\partial^{2} \mathcal{H}}{\partial w \partial \Lambda}(t)\right]_{i} P_{\Lambda}\left(\Lambda^{i}\left(t_{0}\right), t\right)} \\
& P_{w}\left(\Lambda^{i}\left(t_{0}\right), t_{0}\right)=0(n \times n) \text { zero matrix } \\
& P_{\Lambda}\left(\Lambda^{i}\left(t_{0}\right), t_{0}\right)=I(n \times n) \text { identity matrix } \\
& P_{\Lambda}\left(\Lambda^{i}\left(t_{0}\right), t\right)=\left[\begin{array}{cccc}
\frac{\partial \Lambda_{1}(t)}{\partial \Lambda_{1}\left(t_{0}\right)} & \frac{\partial \Lambda_{1}(t)}{\partial \Lambda_{2}\left(t_{0}\right)} & \cdots & \frac{\partial \Lambda_{1}(t)}{\partial \Lambda_{n}\left(t_{0}\right)} \\
\vdots & \vdots & \vdots & \vdots \\
\frac{\partial \Lambda_{n}(t)}{\partial \Lambda_{1}\left(t_{0}\right)} & \frac{\partial \Lambda_{n}(t)}{\partial \Lambda_{2}\left(t_{0}\right)} & \cdots & \frac{\partial \Lambda_{n}(t)}{\partial \Lambda_{n}\left(t_{0}\right)}
\end{array}\right]_{\Lambda^{i}\left(t_{0}\right)} \\
& P_{w}\left(\Lambda^{i}\left(t_{0}\right), t\right)=\left[\begin{array}{cccc}
\frac{\partial \mathrm{w}_{1}(t)}{\partial \Lambda_{1}\left(t_{0}\right)} & \frac{\partial \mathrm{w}_{1}(t)}{\partial \Lambda_{2}\left(t_{0}\right)} & \cdots & \frac{\partial \mathrm{w}_{1}(t)}{\partial \Lambda_{n}\left(t_{0}\right)} \\
\vdots & \vdots & \vdots & \vdots \\
\frac{\partial w_{n}(t)}{\partial \Lambda_{1}\left(t_{0}\right)} & \frac{\partial \mathrm{w}_{n}(t)}{\partial \Lambda_{2}\left(t_{0}\right)} & \cdots & \frac{\partial \mathrm{w}_{n}(t)}{\partial \Lambda_{n}\left(t_{0}\right)}
\end{array}\right]_{\Lambda^{i}\left(t_{0}\right)}
\end{aligned}
$$

4. Examine whether the termination criterion $\left\|\Lambda^{i}\left(t_{f}\right)\right\|$ is satisfied. If that's the case, run $\boldsymbol{\Lambda}^{\boldsymbol{i}}\left(\boldsymbol{t}_{\mathbf{0}}\right)$ one more time to reintegrate the state and costate equations and graph the optimal trajectory and control. If the stopping criterion is not met, use the iteration equation to find the value for $\boldsymbol{\Lambda}^{(\boldsymbol{i}+\mathbf{1})}\left(\boldsymbol{t}_{\mathbf{0}}\right)$, then go to step 3 .

\section{Simulation Study}

We consider the controlled model below

(51)

$$
\frac{\partial z}{\partial t}=\Delta z+\chi_{\omega} u(t)(z-\xi(x, t))
$$

subject to

$$
\begin{aligned}
& z(x, 0)=\sin (\pi x), \\
& z(0, t)=0, z(l, t)=0 .
\end{aligned}
$$

With a diffusivity control parameter $\boldsymbol{u}(\boldsymbol{t})$, the examined model is used to characterize the heat conduction. Coolant flow rate is a diffusivity control variable in the heat transfer process, whereas temperature is a state variable. From $\boldsymbol{x}=\mathbf{0}$ to $\boldsymbol{x}=\boldsymbol{l}$, a e-ISSN: 2148-2683 diffusivity control is applied to a uniform rod of length with nonuniform temperature lying on the $\boldsymbol{x}$-axis. A finite dimensional approximation $\boldsymbol{y}(\boldsymbol{x}, \boldsymbol{t})$ of $\boldsymbol{z}(\boldsymbol{x}, \boldsymbol{t})$ based on the modal space expansion approach is used to simulate the system composed by the nonlinear two-point boundary value problem and proposed diffusivity control.

The state equation can be expanded by a series of orthonormal basis functions $y(x, t) \approx \sum_{k=1}^{n} \boldsymbol{\psi}_{k}(x) \boldsymbol{w}_{\boldsymbol{k}}(t)$, where $\boldsymbol{\psi}_{\boldsymbol{k}}(\boldsymbol{x})=\sqrt{2} \sin (\boldsymbol{k} \pi x)$, which satisfy the homogeneous boundary conditions. The following finite dimensional system is obtained

$$
\frac{d w}{d t}=M w+N w u+K u+L
$$

with initial condition $\mathbf{z}(\mathbf{0})=\frac{\sqrt{2}}{\mathbf{2}}$. The performance index to be minimized is

$$
J=\frac{1}{2}\left[w\left(t_{f}\right)\right]^{T} P\left[w\left(t_{f}\right)\right]+\frac{1}{2} \int_{t_{0}}^{t_{f}}\left(w^{T} R w+u^{T} S u\right) d t
$$

where $\mathrm{P}, \mathrm{R}$ and $\mathrm{S}$ are symmetric positive semi-definite matrices. We simulate the system Eqs. (1)-(3) over $\boldsymbol{t}_{\mathbf{0}}=\mathbf{0} \leq \boldsymbol{t} \leq$ $\boldsymbol{t}_{\boldsymbol{f}}=\mathbf{1}$ with following parameters: $\xi(x, t)=\mathbf{0}, \boldsymbol{j}=\boldsymbol{k}=\mathbf{1}$, $\psi_{k}(x)=\sqrt{2} \sin (k \pi x)$.

\section{Results and Discussion}

Computer codes produced in MATLAB is used for the solution of the system. The boundary conditions for integrating differential equations are $\boldsymbol{P}_{\boldsymbol{\Lambda}}(\mathbf{0})=\boldsymbol{I}$ and $\boldsymbol{P}_{\boldsymbol{w}}(\mathbf{0})=\mathbf{0}$. The initial state $\boldsymbol{w}(\mathbf{0})=\mathbf{0}$ and initial costate $\boldsymbol{\Lambda}(\mathbf{0})=\boldsymbol{\Lambda}^{\boldsymbol{i}}(\mathbf{0})$ are used. The initial guess applied to start the iterative procedure is $\boldsymbol{\Lambda}^{\mathbf{0}}(\mathbf{0})=\mathbf{0}$ and

$$
\left|\Lambda^{1}(1)\right|+\left|\Lambda^{2}(1)\right| \leq 10^{-5}
$$

is the norm used as a stopping criterion. The values of the performance index as a function of the number of iterations are shown in Figure 1. The performance index decreases significantly after 43 iterations, but only slightly improves after the remaining 57 iterations.

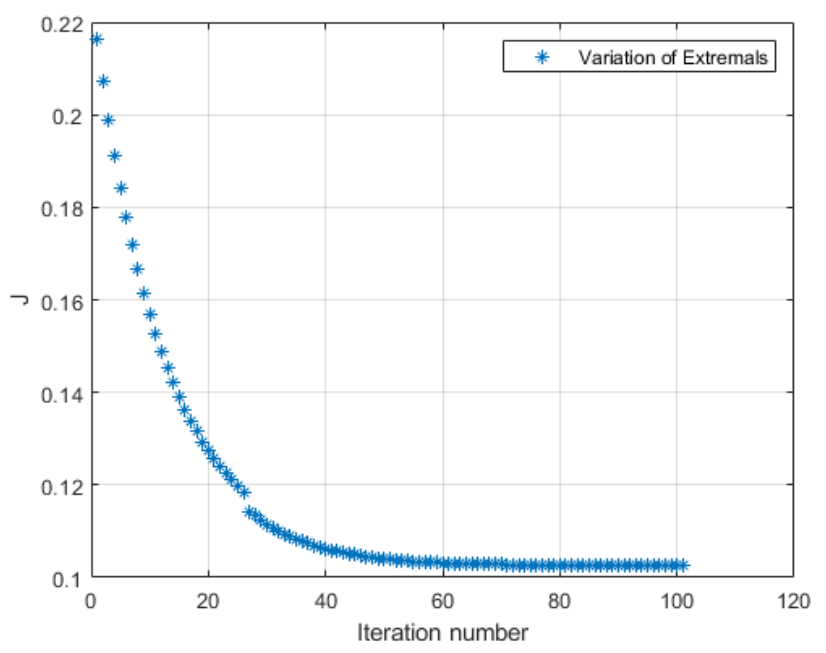

Figure 1. Performance index reduction with the variation of extremals method. 
Temperature profiles in time $\boldsymbol{z}\left(\boldsymbol{x}_{\mathbf{0}}, \boldsymbol{t}\right)$ are curves in the $z t$ plane. Figure 2 shows a comparison of temperature profiles over time with a fixed value of $\boldsymbol{x}_{\mathbf{0}}=\mathbf{0 . 5}$ for uncontrolled and controlled systems using the variation of extremals process.

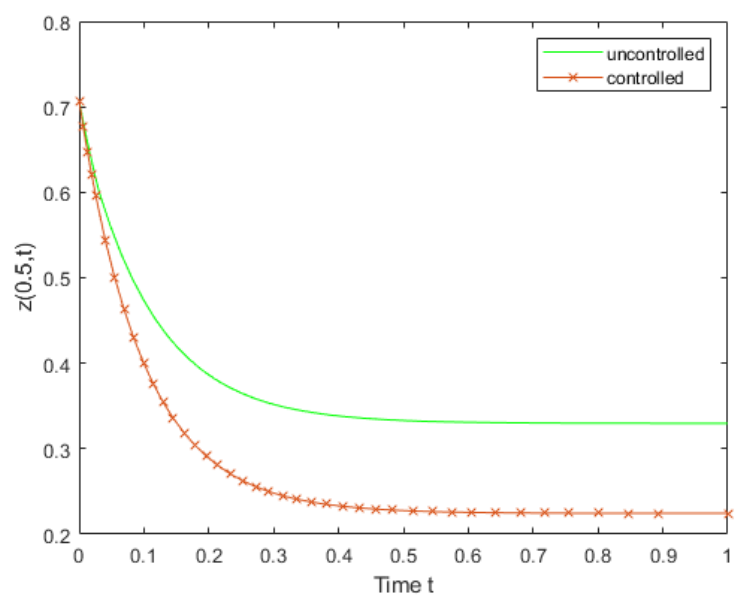

Figure 2. Comparison of the temperature profiles in time at $\boldsymbol{x}_{\mathbf{0}}=\mathbf{0 . 5}$ for uncontrolled and controlled case.

\section{Conclusions and Recommendations}

It is investigated how to control a parabolic PDE with a diffusivity control optimally. The controllability and uniqueness of the system's solution is discussed. A lumped parameter system is created using reduced order modeling. Pontryagin's maximum principle is used to obtain the optimal control function that leads to a nonlinear two-point boundary value problem. An iterative numerical technique, variation of extremals, for determining optimal trajectories and optimal control of the system is discussed. The programming is achieved using computer codes produced in MATLAB. The usefulness and applicability of the proposed strategy are demonstrated through numerical simulation studies. The optimal diffusivity control for different models can be designed by adopting the presented control algorithms in this study. In addition, the system can be controlled by adding boundary control and interior control as well as diffusivity control.

\section{References}

Guliyev, H. F., \& Jabbarova, K. S. (2010). The exact controllability problem for the second order linear hyperbolic equation. Differential Equations and Control Processes, 3, 10-19.

Khapalov, A. Y. (2003). Controllability of the semilinear parabolic equation governed by a multiplicative control in the reaction term: A qualitative approach. SIAM journal on control and optimization, 41(6), 1886-1900.

Kirk, D. E. (2004). Optimal control theory: an introduction. New York: Dover Publications.

Korpeoglu, S. G., \& Kucuk, I. (2018, August). Optimal control of a bilinear system with a quadratic cost functional. In Fourth International Conference on Computing Communication Control and Automation (ICCUBEA) (pp. 1-6).

Lin, P., Leid, P., \& Gao, H. (2007). Bilinear control system with the reaction-diffusion term satisfying Newton's law. ZAMM-

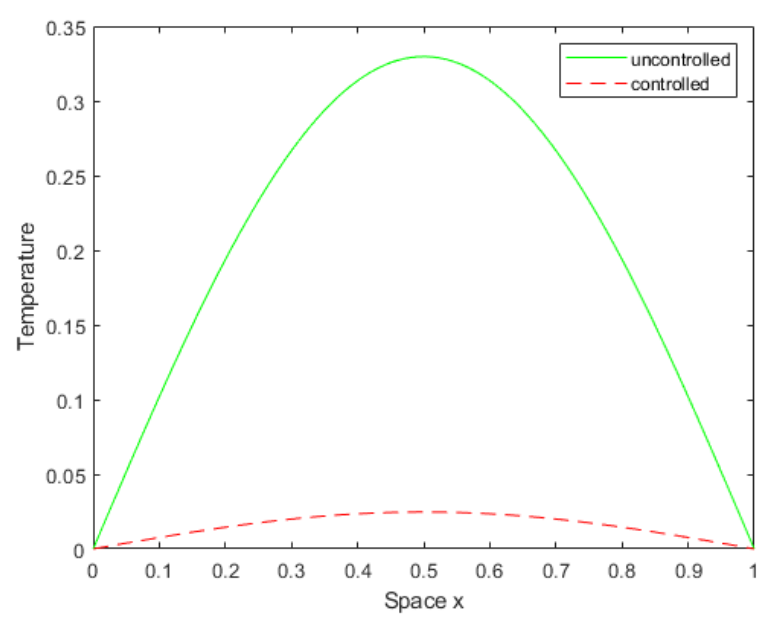

Figure 3. Comparison of the spatial temperature profiles for uncontrolled and controlled case.

Figure 3 depicts the spatial temperature profile of the uncontrolled and controlled systems using the the variation of extremals method at the final time $\boldsymbol{t}_{\boldsymbol{f}}=\mathbf{1}$. Figure 3 shows that at terminal time $\boldsymbol{t}_{\boldsymbol{f}}$, the middle of the rod, which is the position of the highest temperature, cools more slowly for the uncontrolled model than for the controlled model.

Journal of Applied Mathematics and Mechanics, 87(1), 1423.

Lin, P., Gao, H., \& Liu, X. (2007). Some results on controllability of a nonlinear degenerate parabolic system by bilinear control. Journal of mathematical analysis and applications, 326(2), 1149-1160.

Pedersen, M. (1999). Functional analysis in applied mathematics and engineering. USA: CRC press.

Pontryagin, L. S. (1959). Optimal regulation processes. Uspekhi Matematicheskikh Nauk, 14(1), 3-20.

Xu, C., Ou, Y., \& Schuster, E. (2007, December). POD-based reduced order optimal control of parabolic PDE systems via diffusivity-interior-boundary actuation. In 46th IEEE Conference on Decision and Control (pp. 3519-3524).

$\mathrm{Xu}, \mathrm{C} ., \mathrm{Ou}, \mathrm{Y} .$, \& Schuster, E. (2011). Sequential linear quadratic control of bilinear parabolic PDEs based on POD model reduction. Automatica, 47(2), 418-426. 